\title{
On the Evaluation Method of Autonomous Learning Ability of the Chinese and Foreign Students
}

\author{
JiSheng He, Zhijuan Liu*, JiaMing Zhong \\ XiangNan University, Chenzhou Hunan China 423000 \\ hulixue22@163.com;xnupaper@163.com \\ * corresponding author
}

\begin{abstract}
Chinese and foreign scholars have done a lot of research on the evaluation method of students' autonomous learning ability. When the autonomous learning is taken as an ability to evaluate, there are mainly three kinds of evaluation methods: questionnaires, interviews and teacher assessment. When the autonomous learning is taken as a process of learning to study, there are mainly four kinds of evaluation methods: thinking aloud evaluation method, error detection method, trace analysis method and the method of behavior observation. Analyzing the evaluation method of the Chinese and foreign students' autonomous learning ability has important significance both for theoretical and applied research, and for evaluating the students' autonomous learning ability scientifically and reasonably.
\end{abstract}

Keywords- Chinese and foreign students; autonomous learning ability; evaluation method

Chinese and foreign scholars have done a lot of research on the evaluation of students' autonomous learning ability. There is a problem that evaluating students' autonomous learning ability whether engage in the theory or in the application of students' autonomous learning ability. The theory researchers need measurement results of the autonomous learning ability to verify their theoretical analysis, especially the analysis of the composition of the autonomous learning ability. The application research illustrates the effects of educational interventions through the measurement of autonomous learning ability. It can also identify the individual differences of students' autonomous learning ability so that teachers can teach students in accordance with their aptitude. This paper analyzes the evaluation method of the Chinese and foreign students' autonomous learning ability, which has important significance both for theoretical and applied research, and for evaluating the students' autonomous learning ability scientifically and reasonably.

At present, Chinese and foreign researchers mainly adopts two kinds methods to evaluate the ability of autonomous learning. One regards it as a kind of ability and the other regard it as an event or activity or a learning process. The researchers adopt different evaluation methods according to their different aspects.

\section{THE EVALUATION METHOD REGARDING THE AUTONOMOUS LEARNING AS AN ABILITY}

When evaluating the autonomous learning as ability, it is needed to clarify the composition of autonomous learning ability at first. Secondly, it is necessary to make it clear who are the evaluation subjects. Usually the evaluation subjects are teachers, students' parents, students and students' peers. Therefore, the evaluation tools include the teachers' rating scale, parents' evaluation scale, students' self rating scale (self-report questionnaire) and peers' assessment scale. The three kinds of evaluation methods are analyzed commonly used both at home and abroad: questionnaire, interview and teachers' evaluation.

\section{A. Questionnaire}

Questionnaire is a method which uses unified, strictly designed questionnaire to measure individual's psychological characteristics or behavior attitude. Because of it's relatively easy to design, survey and score, questionnaire is the most commonly used method currently in the evaluation of autonomous learning ability

As to the evaluation tool, the most representative and commonly used assessment tools are the "learning and study strategies' investigation" developed by Weinstein et al. and (LASSI,Weinstein et al.,1987), the "learning motivation strategy questionnaire" developed by Pintrich et al. (MSLQ,Pintrich et al,1991). As to the survey of the content, currently both Chinese and foreign researches concentrated on the following areas: (1) Intrinsic motivation factors, including interest, values, self-efficacy, goal orientation, etc.; (2) The use of cognitive strategies, including attention strategy, repetition strategy, organizational strategy, finishing strategy, comprehension strategy, choice points and critical thinking etc.; (3) Metacognitive ability, including time, self management ability, self regulation ability of emotion and self detection and evaluation ability etc. (4) The ability of making use of resources consists of referring to and using information, data and other material resources and seeking academic help etc. As to the questionnaire survey way, self-report test is mainly used. The self-report questionnaire generally involves three aspects. (1) compile or collect test items, and then analyze the study subjects' reaction to the sample and study the degree of concordance between the study subjects and form maker . 
(2) Report the reliability coefficient of the total scale and each sub scale, among which the internal consistency coefficient is generally used. (3) Report the scores of the total scale and subscales relating to the scores of learning achievement test

Several advantages of using self-report questionnaire to measure students' autonomous learning ability tend: (1) Objective, unified, high efficiency, and carrying out with a group. (2) Highly quantitative and standardized statistical results . (3) Low cost and no needs to spend a lot of effort in personnel training. (4) The questionnaire is anonymous, which makes subjects reflect their own opinions more open and realistically .

However, there are also disadvantages in measuring the students' autonomous learning ability with self-report questionnaire. Most questionnaires demand the subject to answer questions structurely, which makes people unable to fully explain their point of view and attitude. At the same time, the effect of questionnaire survey can be affected by subjects' reading comprehension level. Students of different ages have different understanding of the test itself, so the results will be biased.

Chinese, Dong Qi et al (1994) have developed "self monitoring scale" focusing on self monitoring learning ability. "Primary and Secondary School Students' Learning Autonomy Scale" designed by Pang Weiguo (2000) was developed on the basis of the essence, the structure and composition of autonomous learning. It has high reliability and validity in the practical application so that it can measure primary and middle school students' learning autonomy ability on the whole ${ }^{[1]}$.

As for the time management, there are a lot of time management measurement tools developed by foreign scholars, such as Time Structure Questionnaire ${ }^{[2]}$, Time Management Behavior Scale ${ }^{[3]}$ 、 Time Management Questionnaire $^{[4]}$ etc. Huang Xiting, a Chinese scholar, proposed that individual's stable characteristic in time management is a kind of personality, which was called time management disposition. $\mathrm{He}$ also compiled Adolescence Time Management Disposition Scale based on the three-dimensional structure model ${ }^{[5]}$. Zhang Feng did detailed and in-depth study on Huang Xiting's time monitoring subscale, a part of time management disposition scale. Taking middle school students as research objects, he compiled a self monitoring scale suitable for Chinese middle school students. ${ }^{[6]}$ According to different degrees of abstraction of the psychological structure, he divided the self control of time management into two levels: overall monitoring and specific monitoring. The overall monitoring reflects the general characteristics of the individual's self control of time management, which is stable but relatively vague and abstract. The whole monitoring system includes three dimensions: the initiative, openness and effectiveness . The specific monitoring reflects the specific level of individual's self control of time management and the actual operation process of individual's time management and monitoring. The monitoring system includes goal setting, time planning, flexibility, self-control, inspection and evaluation of five dimensions. The formal scale consists of 40 items, including 8 reverse problems, which is suitable in number and easy to understand. The scale has good reliability and validity and can be used as effective tools for the evaluation of students' time management .

In terms of students' self evaluation, questions about the students' plan, inspection and evaluation in the learning process designed by American professor Peg Ertmer from Purdue University can be used for students to evaluate their ability of autonomous learning.

\section{B. Interview}

The interview is a method through which the researcher collects data from the objects and then studies their relevant psychological and behavior characteristics. According to the limited degree to the problems and the ways of answering, interviews can be divided into structured interviews and unstructured interviews. Structured interview refers to a strictly regulate interview, in which the project and the probable reaction of the interview is determine ahead. Non structured interview is a kind of informal interview, in which questions are free to ask and answer.

In the evaluation of autonomous learning ability, currently structured interview is mainly adopted. It generally requires researchers to determine the dimensions or situation of the autonomous learning ahead of time, to design related problems and analysis the content of the interview. For example, "autonomous learning interview schedule" designed by Zimmermann and Martinez - Pons (SRLIS, Zimmerman \& Matinez-Pons, 1986, 1988, 1990) described 8 different learning situations: classroom learning situation, finishing writing homework, finishing math homework, checking scientist homework, checking English homework, reviewing situation preparing test, and the arrangement of learning places when unwilling to complete homework. Students are questioned about their learning methods in each context. Here are some of the questions "The teachers hope that students do math homework correctly. Most of these assignments must be completed without the help of the teacher. When you don't understand math problems at home, do you have any specific measures? When you meet a very difficult math problem, what will you do?"

Chinese and English scholars generally use two methods of analysis to analyze students' description or answer. One is the "bottom-up" analysis, that is, a method of classification and evaluation which based on the analysis of the received data about students' autonomous learning. The second is the "top-down" analysis, which is also called analysis of theoretical guidance. It first classifies students' probable description about autonomous learning, then quantifies and classifies the material obtained according to predetermined type and analyzes the characteristics of students' autonomous learning ability.

\section{Teachers' evaluation method}

In daily communication with students and interactive teaching and studying, teachers have their unique advantage in the evaluation of students' autonomous learning ability. Generally speaking, teachers can more accurately evaluate the students' autonomous learning based on long-term observation of students' learning. Teachers' evaluation can reflect the students' autonomous learning ability to some extent. So it is emphasized both 
at home and abroad.

The evaluation tool adopted in teachers' evaluation method is 'students' autonomous learning evaluation results: scale used by teachers ". The information about students' autonomous learning ability collected through the evaluation of teachers has high reliability. So teachers' evaluation is often regarded as a standard evaluation method in the study of students' autonomous learning ability. The teachers' evaluation is an evaluation of many students under the same system, so it has a stable and consistent standard. The evaluation is in the process of comparing with other students, which makes the evaluation results have the attribute reference norm which is suitable for measurement of different autonomous learning ability, such as individual differences, gender differences and age differences.

In adopting teachers' evaluation method, teachers are required to have a comprehensive understanding of students' learning ability, otherwise the evaluation results may not be reliable. In addition, teachers' high quality is required. If there are more than two teachers, the consistency between the different evaluation results is required.

\section{AUTONOMOUS LEARNING AS A LEARNING PROCESS}

If autonomous learning is regarded as a learning process or activity, investigate the dynamic learning process of students' autonomous learning ability. Four kinds of evaluation methods are adopted at home and abroad when studying autonomous learning as a process of learning: thinking aloud evaluation method, error detection method, trace analysis method and the method of behavior observation.

\section{A. Thinking aloud evaluation method}

Thinking aloud evaluation refers to a method students speaking out their thoughts and cognitive processes while engaging in a learning activity, during which the learning process are analyzed and evaluated. In the process of thinking aloud test, students are generally required to speak out their thinking process not psychological activities, in order not to influence their normal activities of learning.

The evaluation technique is often used in the study of reading autonomous learning, which is mainly used to detect whether the students master the learning strategies and problems solving steps. The advantage of this measurement method is that it can accurately reflect the quality of individual's learning strategies and processes. The disadvantage is that it requires the subjects to share part of their energy to describe their inner activities, which will affect the autonomous learning process, so that the results of the evaluation have slight deviation.

\section{B. Error detection method}

Metacognitive monitoring, which identifies the degree of correspondence between target and current task state, is a prerequisite process of autonomous learning and self studying adjustment. The cognitive evaluation caused by metacognitive monitoring plays a guiding role on further task completion steps, which is also the base of metacognitive monitoring. Therefore, in the study of autonomous learning, evaluation of metacognitive monitoring is a great concern to researchers.

In order to measure the metacognition monitoring in which the students are regarded as prerequisite elements of learning control, researchers sometimes put some errors in the students' learning materials or tasks and then observed: (1) whether they noticed these errors; (2) what do they do when they noticed the error. (This method is called error detection method.)

In error detection, researchers mainly focus on changes of the situation that students report errors to investigate the students' autonomous learning ability. The error situation can be divided into two categories. One is to tell students that materials contain errors before or after the students' learning. The other one is not to tell the students that learning materials contain errors. The latter is more close to students' daily learning situation, because in this situation students often believe that their own learning content is correct, with no intentionally settled error. The key feature shown in these two situations is material containing information which tells the students about the nature of the error. There are also several forms of error monitoring indexes in students' materials, including the place with marked wrong and the place that correct mistakes, etc. If students have difficulties in understanding learning materials, they can just mark the wrong parts from correct parts.

\section{Trace analysis method}

Trace analysis method refers to a method that analyzes "marks" left by students while they are engaged in learning tasks, such as underlines, annotations, notes and so on, through which researchers can infer the learning strategies and effects of the students. For example, students often use the underlines while reading books to indicate that the underlined contents are different from other contents. So it can be inferred that the underlined content is considered as an important one or one needs to be further understood. When students copy down information from their textbooks or other sources, they are considered as repeating the information; When students write down such words in their notebook or contact book : "ask for other students' help ", they are considered as trusting and regarding others as a resource to help themselves to solve the current task; When students summarize or make analogy of what they learned, they are considered to organize information into a larger unit; When students make such notes beside their homework: after finishing homework, what should be done next? It reflects their strategic plan.

Trace analysis method can help the researcher to speculate the autonomous learning strategies used by the students. But using this method requires a lot of time, because first the researcher needs to find out a lot of information which can reflect the students' learning process and learning strategies, and then systematically summary it, classify it , and analyze it.

\section{Behavior observation method}

Behavior observation refers to a method to inspect the individual's external behavior performance or activities in a certain period of time, and explore the characteristics and rules of students' mental activities. Using this evaluation method to study autonomous learning has 
three advantages: (1) reflecting what the students did , what they recalled and what they believed; (2) linking students' behavior with task conditions, especially those task conditions that can give feedback; (3) compensating for deficiencies of other measurements of the autonomous learning ability.

Turner (Turner, 1995) did researches on how classroom reading task influence the middle school students' reading strategies and learning strategies, their persistence, and their mind controlling through the observation of students' autonomous learning activities. The observation process can be divided into three parts: Firstly researchers recorded identified materials, including the observed student's name, class, date and time; Secondly, the observer records students' behavior and teaching situation on the spot; Finally, the observer uses a checklist including 5 types of reading strategy to evaluate students' condition of grasping reading strategies. These 5 kinds of reading strategies are decoded strategies (eg. the use of context clues), comprehension strategies (eg. prediction, summary), general learning strategies (eg. retelling, finishing, organization), volitional control strategies (eg. resisting interference) and persistence strategies (eg. finishing the difficult task). They collect relevant information on autonomous learning by time samplings. Usually they take three minutes as a unit to observe and record the students' meeting with the expected autonomous learning events. In the observation process, the observer sits next to the observed students, recording the students' behavior, facial expressions, eye movements, finger content and writing and so on in the reading process. After each observation, the observer will talk with the students about the task completed just now. The conversation contains four open questions which are used to evaluate students' self-regulated learning in two key aspects: the intention to complete specific tasks and the thoughts the students have in finishing the task. This method shows its high reliability and validity, and is worthy of reference for the study of autonomous learning ability.

As to the above two main types of evaluation methods of the autonomous learning ability, which specific evaluation methods should be chosen in the actual teaching and researching, depends on the specific situation. Essentially, autonomous learning is not only a kind of event or activity but also a kind of ability. Therefore, these two types of evaluation methods can be used in combination.

\section{ACKNOWLEDGEMENTS}

The study has obtained Hunan Province Education Science "the 12th 5-year plan" programming research project (XJK012BJJ002、XJK012CGD032).

\section{REFERENCES}

[1] Pang weiguo.The autonomous learning - the principle and strategy of learning and teaching[M]. Shanghai:East China Normal University press, 2003.

[2]Bond,M,Feather.M.Some correlates of structure and purpose in the use of time[J].Journal of Personality and Social Psychology, 1988,(1):321-329.

[3]Macan,T.H.,Shahani,C.,Dipboye,R.1.,et al.A.P.College students'time managerment : Correlations with academic performance and stress[J].Journal of Educational Psychology, 1990,82(4) :760-768.

[4]Britton,B.K.,Tesser,A..Effect of time- management practices on College grades $[\mathrm{J}]$. Journal of Educational Psycholog, 1991,83(3): $405-410$

[5] Huang Xiting, Zhang Zhijie.Compilation of time management disposition scale for adolescents[J]. Acta Psychologica Sinica, 2001,33(4):338-343.

[6] Zhang Feng.The research on the time management of self controlling scale for middle school students[J]. psychological science, 2007,30 (3):668-671. 